\title{
A HISTORICAL VIEW OF HYBRID SIMULATION/ANALYTIC MODELS
}

\author{
Robert G. Sargent \\ Simulation Research Group \\ 449 Link Hall \\ Syracuse University \\ Syracuse, New York 13244
}

\begin{abstract}
A description of hybrid simulation/analytic models and modeling is presented. Then a historical view of them are given by dividing time into three periods: pre1978, 1978-1984, post-1984. Finally, conclusions are drawn based upon this historical view.
\end{abstract}

\section{INTRODUCTION}

We present a historical view of hybrid simulation/analytic models and modeling (hereafter referred to as hybrid models and modeling). This history will be divided into three time periods: pre-1978, 19781984, and post-1984. The reason for this division is because of the activities regarding hybrid models and hybrid modeling during the 1978-1984 time period including the development of the "unified view" by Shanthikumar and Sargent (1983).

Analytic and simulation models can be considered as two end-points of a spectrum of possible mathematical models that can be used in modeling. An analytic model is a set of equations that can characterize a system or a problem entity. Its solution procedure usually uses either an analytical equation or a numerical algorithm that has been developed for the set of model equations to obtain the desired results. A simulation model is a dynamic or an operating model of a system or problem entity that "mimics" the operating behavior of the system or problem entity and contains its functional relationships. The simulation model is often called the conceptual model (for example, see Sargent (1994)). Its solution procedure consists of running a computerized model of the conceptual model, usually called the simulator, collecting data on its behavior, and analyzing the data to obtain the desired results. Sometimes it is desirable to combine analytic and simulation models (or modeling) into a hybrid model (or modeling) and use them if they are cost efficient. (For further discussion see Shanthikumar and Sargent (1983).)
The remainder of this paper is organized as follows. In Section 2, the definitions of hybrid models and modeling are given along with the classes of hybrid models and usages of hybrid modeling following the work of Shanthikumar and Sargent (1983). Section 3 contains the history of the three time periods and Section 4 contains the conclusions.

\section{HYBRID MODELS AND MODELING}

We first define the difference between hybrid models and hybrid modeling. The combining of analytic and simulation models may be achieved either through the models and their solution procedures or through the use of the solution procedure of independent analytic and simulation models. If such a combination is achieved through the models and their solution procedures, one has a hybrid model. If the solution procedure of independently developed analytic and simulation models of the total system are used together in problem solving, one is performing hybrid modeling. (See Figure 1.) Thus the following definitions:

DEFINITION. A hybrid model is a mathematical model which combines identifiable simulation and analytic models.

DEFINITION:. Hybrid modeling consists of building independent analytic and simulation models of the total system, developing their solution procedures, and using their solution procedures together for problem solving.

Shanthikumar and Sargent (1983) presented four classes of hybrid models and examples of each. The four classes are as follows:

CLASS I. A model whose behavior over time is obtained by alternating between using independent simulation and analytic models. The simulation (analytic) part of the model is carried out without intermediate use of the analytic (simulation) part (see Figure 1.a). 


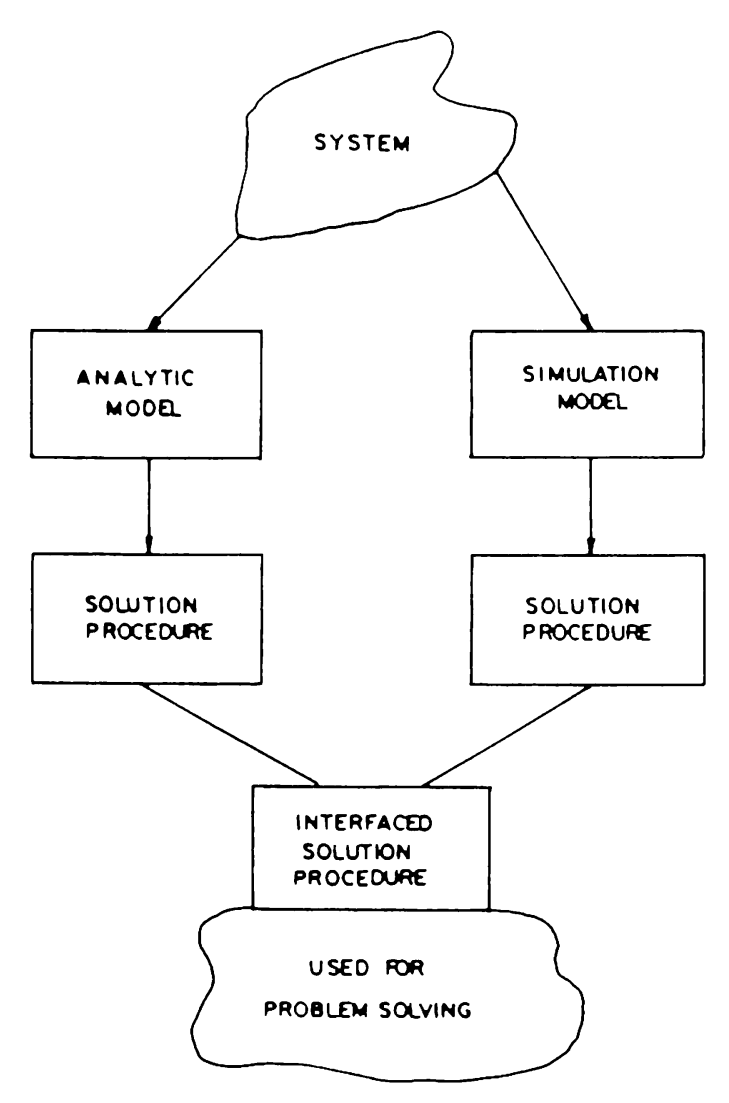

Figure 1.a. Class I hybrid model

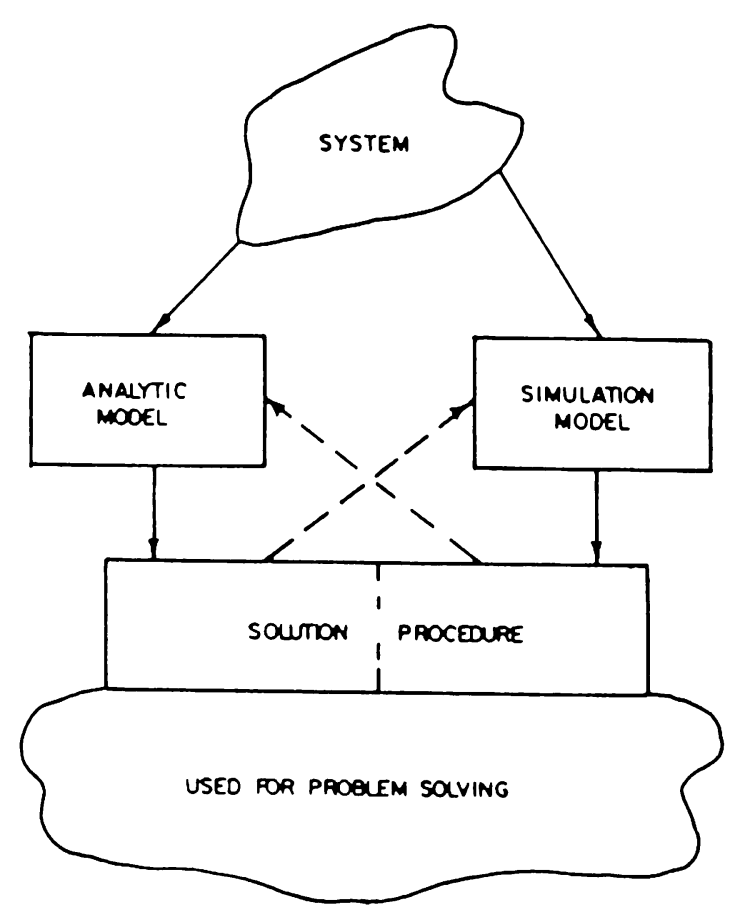

Figure 1.b. Class II hybrid model
CLASS II. A model in which a simulation model and an analytic model operate in parallel over time with interactions through their solution procedure (sce Figure 1.b).

CLASS III. A model in which a simulation model is used in a subordinate way for an analytic model of the total system (see Figure 1.c).

CLASS IV. A model in which a simulation model is used as an overall model of the total system, and it requires values from the solution procedure of an analytic model representing a portion of the system for some or all of its input parameters (see Figure 1.d).

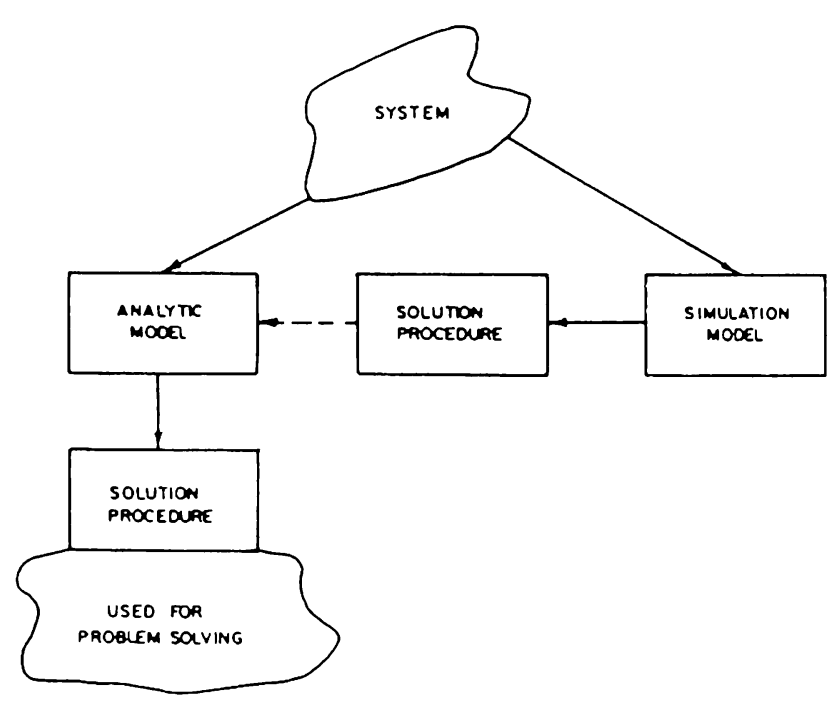

Figure 1.c. Class III hybrid model

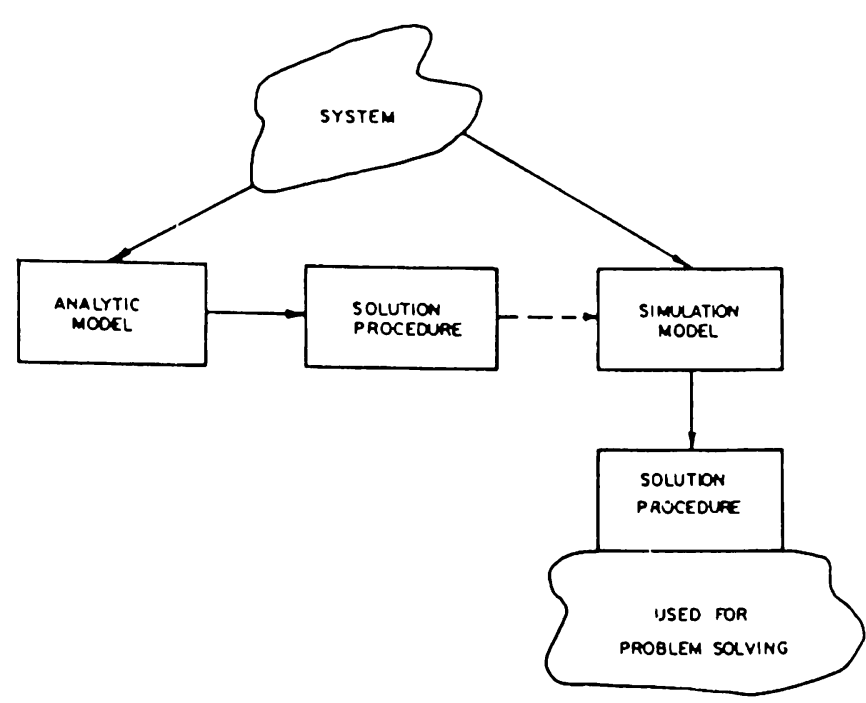

Figure 1.d. Class IV hybrid model 


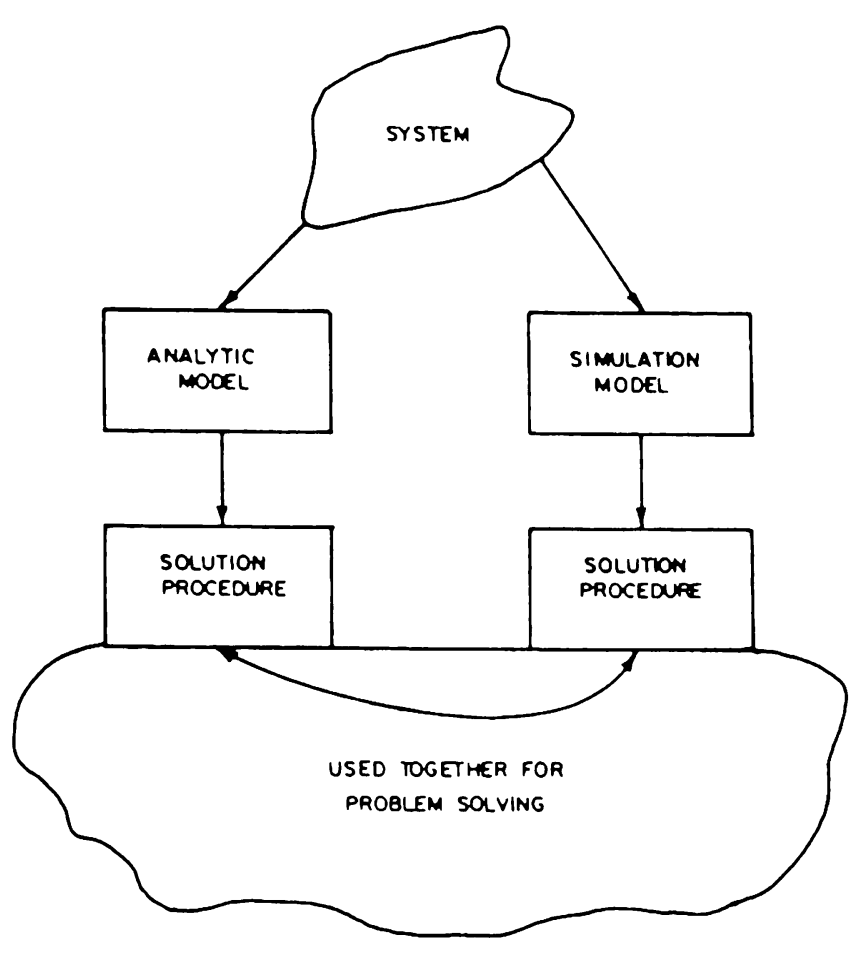

Figure 1.e. Hybrid modeling

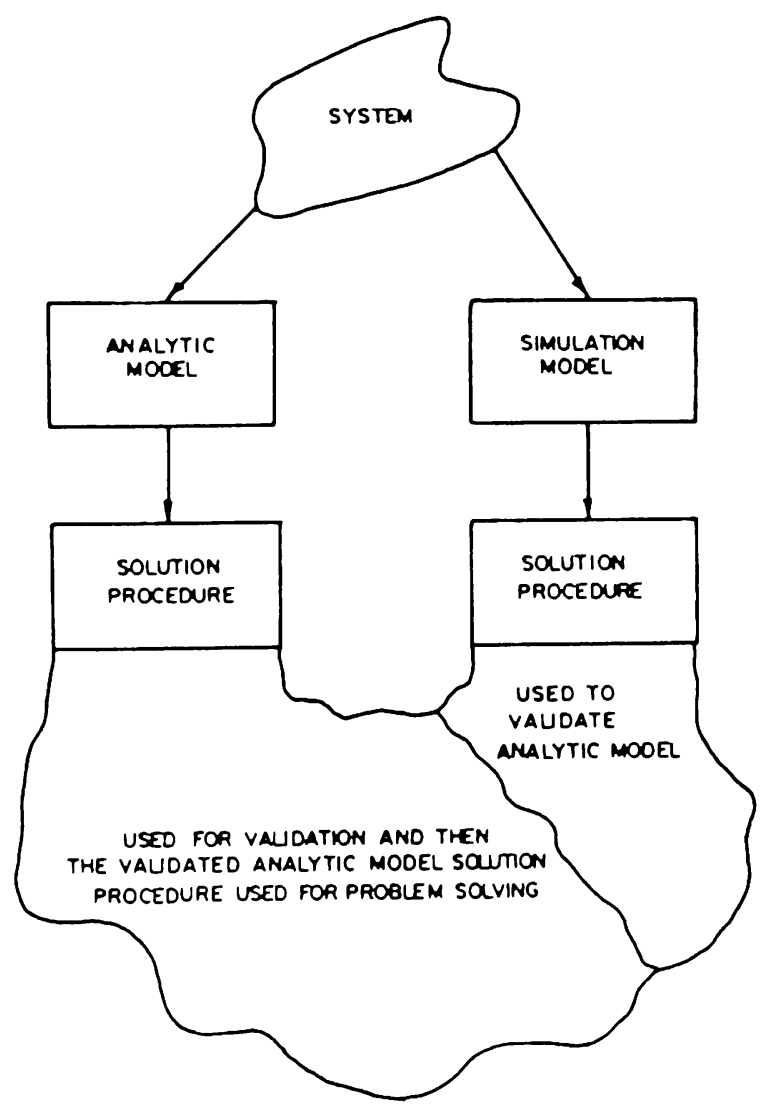

Figure 1.f. Hybrid modcling for validation
Class I and Class II hybrid models depend upon the time dependent behavior of the system. If the time dependent behavior can be completely decomposed so that some part of it can be solved analytically, then one would have a Class I model. If both an analytic model and a simulation model are required to operate in parallel with interactions through the solution procedure with respect to the time dependent behavior, then one has a Class II model.

Class III and Class IV hybrid models use analytic models and simulation models, respectively, as models of the total system. For Class III models, usually a simulation model(s) of either a subsystem(s) or the total system is used to obtain estimates for at least some of the values of the parameters of the analytic model of the total system. Class IV models obtain values for some or all of the simulation model parameters from the solution procedure(s) of an analytic model(s).

Four usages of hybrid modeling (see Figure 1.e) were given in Shanthikumar and Sargent (1983) and they are (1) developing Operations Research/Management Science (OR/MS) theory, (2) gaining insight into system bchavior, (3) validating analytic models (see Figure 1.f), and (4) performing optimization. (See Shanthikumar and Sargent (1983) for some examples.)

\section{HISTORY OF HYBRID MODELS AND MODELING}

To discuss the history of hybrid models and modeling, we will consider the three time periods of pre1978, 1978-1984, and post-1984. These time periods were chosen primarily to determine the effect of "A Unifying View of Hybrid Models and Modeling" by Shanthikumar and Sargent (1983).

In the pre-1978, onc finds in the literature only a few isolated examples of hybrid models and modeling. These were performed by very sophisticated modelers (sce, e.g., Fetter and Thompson (1969)). This author believes this was because of the lack of sophisticated modelers and the state-of-the-art of modeling and computer technology.

During the 1978-1984 time period, one finds a considerable amount of activity in hybrid models and hybrid modeling. (See Shanthikumar and Sargent (1983) for some references.) This consisted of two types of work. One was the application of hybrid models and hybrid modeling to specific problems. The other was promoting and developing "systematic" approaches to hybrid models and modeling. The latter includes such publications as Ignall and Kolesar (1979), Ignall, Kolesar, and Walker (1978), and Shanthikumar and Sargent (1983). In addition, sessions were held on hybrid models and modeling at the 1982 and 1984 Winter 
Simulation Conferences, and seminars were given at universities and talks at ACM chapter meetings by at least this author. It is interesting to note that Ignall and Kolesar (1979) emphasized the need to have increased usage of hybrid modeling and that Shanthikumar and Sargent (1983) stated that the primary purpose of their classification of hybrid models (i.e., the four classes that are given in Section 2 of this paper) was to aid in the development of hybrid models with a secondary purpose of aiding in communication about hybrid models. Furthermore, they emphasized in their conclusions the need for research in hybrid models.

In the post-1984 period, one finds only limited examples of hybrid models and modeling in the literature. Furthermore, these usually do not refer to the systematic approaches or classifications that were developed in the 1978-84 time period. This author is only aware of one book, Operations Management (McClain and Thomas 1985), that refers to the Shanthikumar and Sargent (1983) unifying work and this is just a passing comment. Thus the time period of post-1984 is quire similar to pre-1978 time period except for an increase in validating analytic models using simulation models which is one usage of hybrid modeling.

\section{CONCLUSIONS}

This author has concluded the following from his historical review of hybrid (simulation/analytic) models and modeling:

(1) The usage of hybrid models and modeling is currently quite limited.

(1) The systematic approaches and classifications of the 1978-84 time period has not been incorporated into the field.

(3) Essentially no research is currently occurring in hybrid models.

(4) Hybrid models and modeling are highly useful.

(5) Research is needed in this field.

One can ask why conclusions (1) and (2) have occurred after the time period of 1978-84; in particularly with the development of computer technology and software systems for OR/MS (including simulation systems). This author believes this has occurred primarily because this "material" is not included in textbooks and is not taught in the classroom and secondarily it requires a certain amount of sophistication in modelers. Hopefully, future texts will include this approach to modeling in them.

Research in hybrid models needs to be done and hopefully this will occur in the future. Sessions at conferences such as the Winter Simulation Conference provides visibility to the field which should increase its usage and stimulate research in it.

\section{REFERENCES}

Fetter, F.B., and J.O. Thompson. 1969. A Decision Model for the Design and Operation of a Progressive Patient Care Hospital. Medical Care 7, 450-462.

Ignall, E., and P. Kolesar. 1979. On Using Simulation to Extend OR/MS Theory: The Symbiosis of Simulation and Analysis. In Current Issues in Computer Simulation, N.R. Adams and A. Dogramaci (eds.), Academic Press, New York.

Ignall, E., P. Kolesar and W. Walker. 1978. Using Simulation to Develop and Validate Analytical Models. Opns. Res. 28, 237-253.

McCain, J.O., and L.J. Thomas. 1985. Operations Management. 2nd ed. Prentice-Hall Inc.

Sargent, R.G. 1994. Verification and Validation of Simulation Models. In Proceedings of 1994 Winter Simulation Conference, eds. J.D. Tew, M.S. Marivaianan, DA. Sadowski, A.F. Seila, Orlando, FL. Shanthikumar, J.G., and R.G. Sargent. 1983. A Unifying View of Hybrid Simulation/Analytic Models and Modeling. Operations Research 30, 1030-1052.

\section{AUTHOR BIOGRAPHY}

ROBERT G. SARGENT is a Professor at Syracuse University. He received his education at the University of Michigan. Dr. Sargent has served his profession in numerous ways and has been awarded the TIMS College on Simulation Distinguished Service Award for longstanding exceptional service to the Simulation Community. His research interests include the methodology areas of modeling and discrete event simulation, model validation, and performance evaluation. Professor Sargent is listed in Who's Who in America. 